\title{
Identification of a Human-specific Epitope in a Conserved Region of the La/SS-B Autoantigen
}

\author{
Yi Ma Weng, * Jane McNeilage, * Fiona Topfer, * James McCluskey, ${ }^{\star}$ and Tom Gordon * \\ * Department of Clinical Immunology, Flinders Medical Centre, Bedford Park, South Australia 5042, Australia; \\ and ${ }^{\ddagger}$ Australian Red Cross Society, S.A. Division, Blood Transfusion Service, South Australia 5000 Australia
}

\begin{abstract}
Human anti-La/SS-B autoantibodies are known to react with highly conserved epitopes suggested to be functional or active sites on the La/SS-B polypeptide. This study was designed to determine whether the autoantibodies also react with poorly conserved regions of $\mathrm{La} / \mathrm{SS}-\mathrm{B}$ as predicted by an antigendriven autoimmune response. Binding of human autoantibodies to purified human, mouse, and bovine recombinant fragments representing immunodominant regions of the La/SS-B polypeptide was compared using Western blotting and ELISA. A cross-reactive epitope was located in the highly conserved $\mathrm{NH}_{2}$-terminal region of La/SS-B. Significantly, human-specific epitopes were identified in both the conserved RNA-recognition motif and a poorly conserved $\mathrm{COOH}$-terminal fragment, providing direct evidence for an autoantigen-driven response. The lack of autoantibody cross-reactivity with a conserved domain of mouse and bovine La/SS-B implies that a small number of residues in human autoepitopes may be critical for autoimmunogenicity. (J. Clin. Invest. 1993. 92:1104-1108.) Key words: autoantibody • epitope $\bullet$ recombinant $\bullet$ autoantigen $\bullet$ immunodominant
\end{abstract}

\section{Introduction}

In autoimmune diseases such as Sjögren's syndrome or SLE, the human B cell response to the La/SS-B autoantigen is directed to multiple epitopes that span the full length of the $\mathrm{La} /$ SS-B polypeptide (1-7). An immunodominant, discontinuous epitope has been identified at the $\mathrm{NH}_{2}$ terminus ( $\mathrm{LaA}$; amino acids [aa ] 1 107) and may be recognized early in the response (8). The majority of anti-La/SS-B sera also react with a putative RNA-recognition motif(RRM; aa 112-187) located in the middle region of the molecule ( $\mathrm{LaC}$; aa 111-242) $(1,7)$. The RRM and an $\mathrm{NH}_{2}$-terminal flanking region appear to be necessary for binding to a short sequence of uridylate residues at the

Address correspondence to Tom Gordon, FRACP, FRCPA, Ph.D., Department of Clinical Immunology, Flinders Medical Centre, Bedford Park, South Australia 5042, Australia.

Received for publication 11 January 1993 and in revised form 5 March 1993.

1. Abbreviations used in this paper: aa, amino acid; GST, glutathioneS-transferase; RRM, RNA recognition motif.

J. Clin. Invest.

(C) The American Society for Clinical Investigation, Inc.

0021-9738/93/08/1104/05 \$2.00

Volume 92, August 1993, 1104-1108
3' terminus of small RNAs (mostly RNA polymerase III transcripts) to form a ribonucleoprotein complex $(9,10)$. The majority of sera also react with a $\mathrm{COOH}$-terminal epitope ( $\mathrm{LaL} 2 /$ 3; aa 346-408) that may be recruited later in the anti-La/SS-B response (7). The IgG autoantibodies are present in high serum concentrations (11) and are associated with particular MHC class II alleles (12). The above observations constitute indirect evidence for an antigen-driven mechanism of autoantibody production.

The broad cross-reactivity of human anti-La/SS-B sera with La/SS-B antigen from diverse mammalian species indicates the presence of conserved epitopes (13). Antigenicity of conserved structural elements has been noted as a general rule for nuclear autoantigens (14). Taken together with the observation that many autoantibodies appear capable of inhibiting the cellular functions served by the autoantigens (reviewed in 15), it has been proposed that the anti-La/SS-B response targets highly conserved epitopes that correspond to functional domains of the molecule (13). This pattern of autoantibody specificity contrasts with the species-specific epitopes recognized after immunization with La/SS-B protein (13). The strong reactivity of human autoantibodies with intact La/SS-B antigen from other species has obscured the definition of species-specific autoepitopes. The recent cloning of mouse La/SS$B$ in our laboratory (16) has permitted expression of mouse La/SS-B fragments corresponding to the three major human $\mathrm{La} / \mathrm{SS}-\mathrm{B}$ antigenic regions. To investigate whether the human autoantibody response to La/SS-B targets human-specific determinants, we have compared reactivity of human anti-La/ SS-B sera with individual human, murine, and bovine fragments of La/SS-B. The data confirm and map an epitope shared in human and mouse La/SS-B and located in the conserved $\mathrm{NH}_{2}$-terminal region of the molecule. In addition, a human-specific epitope was located in the poorly conserved $\mathrm{COOH}$-terminal region, but surprisingly, an immunodominant, human-specific epitope was also identified in the conserved RNA binding domain. The findings implicate antigendriven autoimmunity in the mature anti-La/SS-B autoantibody response.

\section{Methods}

Derivation and expression of mouse and bovine DNA encoding La/SS$B$ fragments. Fig. 1 shows alignment of the human and mouse LaA, $\mathrm{LaC}, \mathrm{LaL} 2 / 3$, and RRM regions. The bovine RRM sequence is included in the boxed RRM region. Alignment was configured using the FAST.P align program (17). Four DNA fragments encoding nonoverlapping subregions of the mouse La/SS-B coding sequence were derived by PCR (18). The primers were synthesized based on the mouse 

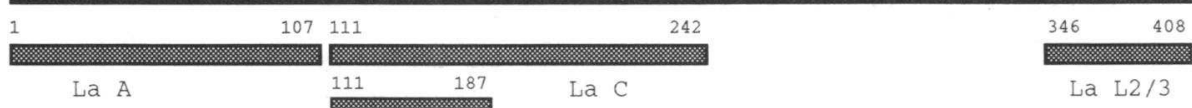

Mouse La

RRM

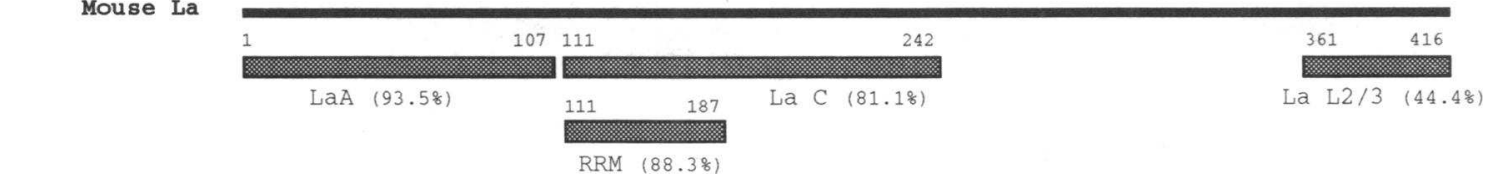

B

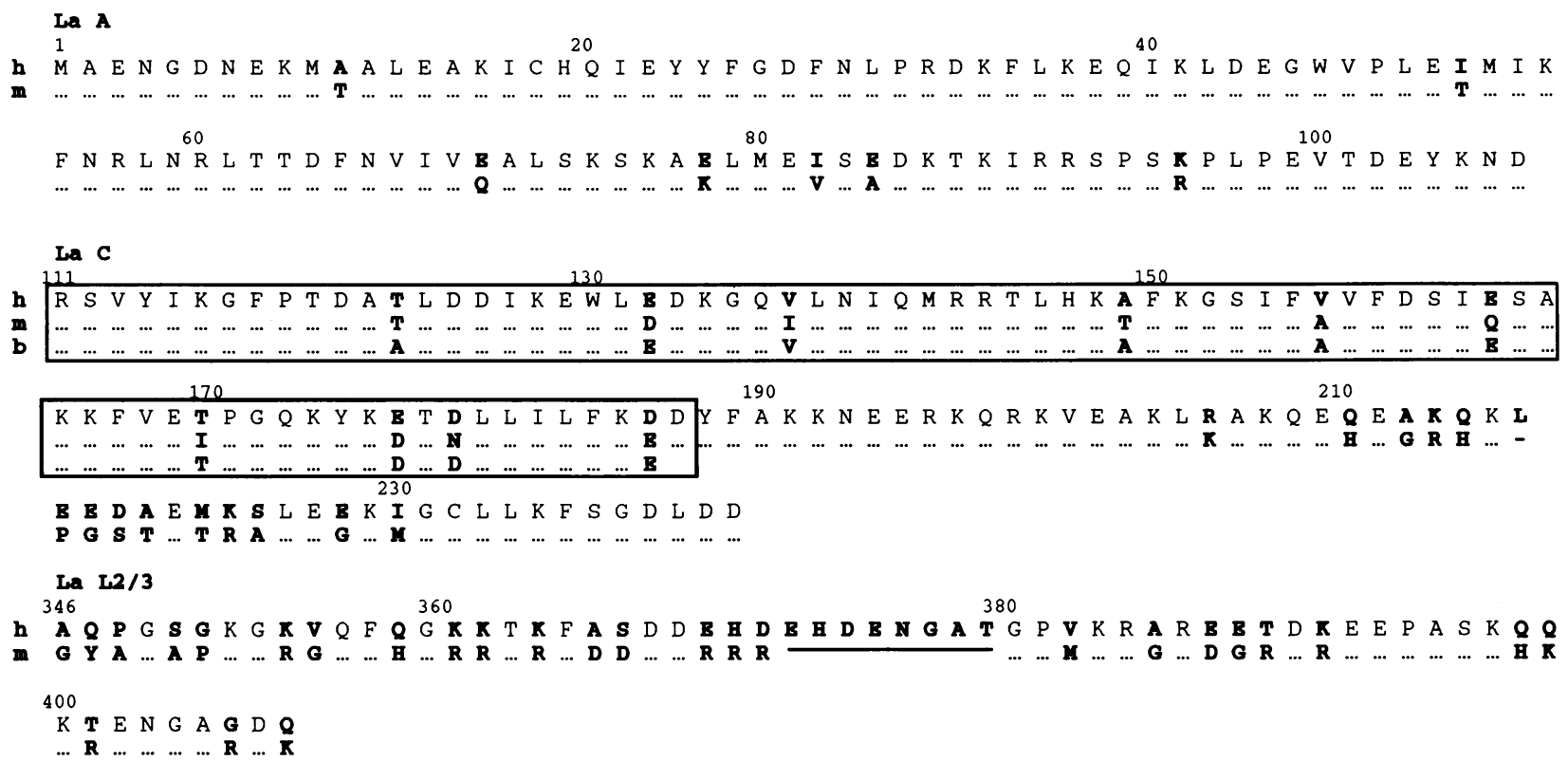

Figure 1. (A) Schematic diagram of the PCR-derived mouse and human La/SS-B fragments subcloned into the pGEX-2 expression vector. The region of the protein encoded by each fragment is given by the aa numbers. The sequence identities of the different regions are indicated in brackets. $(B)$ Comparison of predicted aa sequences of human $(\mathrm{h})$ and mouse $(\mathrm{m}) \mathrm{LaA}, \mathrm{LaC}$, and $\mathrm{LaL} 2 / 3$ fragments. Bold aa indicate differences between the species, and dashes indicate sequence gaps introduced to facilitate alignment. Alignment was configured using the FAST.P align program (15). The bovine (b) RRM sequence is included in the boxed RRM region.

La/SS-B cDNA sequence to correspond to the previously defined fragments $\mathrm{LaA}, \mathrm{LaC}, \mathrm{LaL} 2 / 3$ and the RRM on human $\mathrm{La} / \mathrm{SS}-\mathrm{B}$, with the addition of a BamHI site and an EcoRI site on the 5' and 3' oligonucleotides, respectively. The amplification was performed for 25 cycles, with each cycle comprising $1 \mathrm{~min}$ at $95^{\circ} \mathrm{C}, 2 \mathrm{~min}$ at $55^{\circ} \mathrm{C}$, and $3 \mathrm{~min}$ at $72^{\circ} \mathrm{C}$. Each mouse La/SS-B DNA fragment was subcloned into the pGEX-2 vector, sequenced to confirm in-frame gene fusions and absence of amplification errors as described (7), and expressed as a glutathione-S-transferase (GST) fusion protein. The recombinant proteins were purified from the soluble fraction of lysates of Escherichia coli strain LE 392 transformants prepared with 1\% Triton X-100. The mouse $\mathrm{LaC}$ insert was subcloned into the $\mathrm{pQE}-9$ vector (QIAGEN Inc., Chatsworth, CA), expressed as an in-frame hexa-his-fusion protein with six histidines at the $\mathrm{NH}_{2}$-terminus, and purified as described (19). A PCR DNA fragment encoding the bovine RRM (aa 111-187) was derived from a bovine La/SS-B cDNA (20) (a gift from Dr. Edward Chan, The Scripps Research Institute, La Jolla, CA), subcloned into the pGEX vector and the GST-fusion protein purified as above.

Western blotting. Human and mouse La/SS-B-GST-fusion proteins were separated on $12.5 \%$ SDS-polyacrylamide gels and transferred to nitrocellulose membrane (Bio-Rad Laboratories, Richmond, CA). After transfer, the remaining protein binding sites were blocked for $1 \mathrm{~h}$ at room temperature with a solution of $5 \%$ nonfat dried milk powder in PBS (0.05 $\mathrm{M}$ sodium phosphate, $0.15 \mathrm{M} \mathrm{NaCl}, \mathrm{pH} 7.4$ ). The nitrocellulose was probed with a 1:3,000 dilution of either a polyclonal rabbit anti-GST serum or a pool of 18 anti-La/SS-B precipitin-positive human sera, and binding of the antisera visualized with a horseradish peroxidase-conjugated sheep anti-rabbit or rabbit anti-human IgG (Silenus Labs. Pty, Ltd., Melbourne, Australia) and enhanced chemiluminescence (Amersham International, Amersham, UK).

ELISA. Binding of human anti La/SS-B sera (diluted 1:500) to the recombinant human and mouse $\mathrm{La} / \mathrm{SS}-\mathrm{B}$ fusion proteins was determined by ELISA as previously described with coating concentrations chosen to give equivalent $\mathrm{OD}_{405 \mathrm{~nm}}$ readings with the rabbit anti-GST serum $(8,21)$. Results were expressed in $\mathrm{OD}_{405 \mathrm{~nm}}$ units as the mean of duplicates. Competition ELISAs were performed as described (8) with percent inhibition calculated as $\left(\mathrm{OD}_{405}\right.$ without inhibitor $-\mathrm{OD}_{405}$ with inhibitor $) /\left(\mathrm{OD}_{405}\right.$ without inhibitor $) \times 100$.

Human sera. The 18 human anti-La/SS-B positive sera used in the study were positive by counterimmunoelectrophoresis and reacted with recombinant human La/SS-B by ELISA. Control sera were obtained from 15 healthy individuals.

\section{Results}

Reactivity of human anti-La/SS-B-positive sera with human, mouse, and bovine La/SS-B fusion proteins. The fusion proteins expressed by the human and mouse La/SS-B subclones were purified and initially identified by immunoblotting with a 


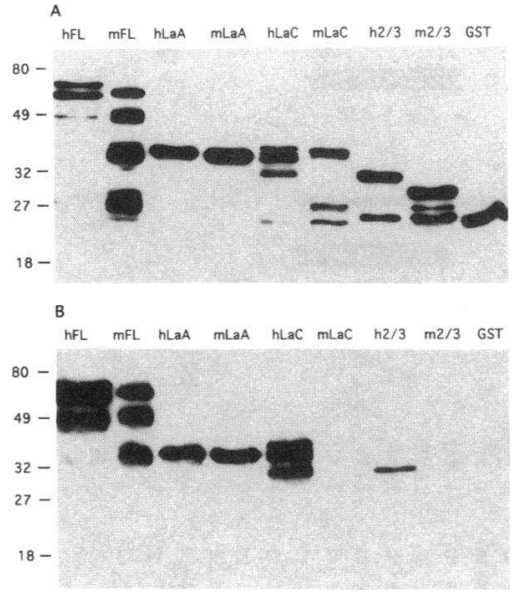

Figure 2. Western blots of human $(h)$ and mouse $(\mathrm{m})$ full-length (FL) La, LaA, LaC and LaL2/3 GST fusion proteins probed with (A) a polyclonal rabbit anti-GST serum and (B) pooled human antiLa/SS-B sera. The human antiserum fails to cross-react with either $\mathrm{mLaC}$ or $\mathrm{mLaL} 2 / 3$. rabbit anti-GST serum (Fig. $2 A$ ). The eight recombinant La/ SS-B proteins were of the expected molecular weight by gel electrophoresis. Bands corresponding to proteins of lower $M_{\mathrm{r}}$ were presumed to represent degradation products (7). A representative Western blot of the human and mouse recombinant La/SS-B proteins probed with the pooled human anti-La/SS$B$ sera is shown in Fig. $2 B$. The human antisera reacted with the full-length La/SS-B and LaA from both species, together with human $\mathrm{LaC}$ and $\mathrm{LaL} 2 / 3$, but failed to react with mouse $\mathrm{LaC}$ and $\mathrm{LaL} 2 / 3$. The same result was obtained when the West-

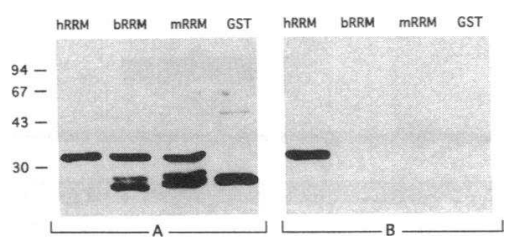

Figure 3. Western blots of human $(\mathrm{h})$, bovine (b), and mouse (m) GSTfusion proteins encoding the RRM probed with $(A)$ a polyclonal rabbit anti-GST serum and $(B)$ pooled human anti-La/SS-B sera.

ern blots were probed with $1: 100$ and 1:1,000 dilutions of antiserum (data not shown). No reactivity was noted with a hexahis-mouse LaC fusion protein expressed in the $\mathrm{pQE}$ vector, indicating that the lack of reactivity with GST mouse $\mathrm{LaC}$ was not due to a masking effect of the GST moiety (data not shown). Fig. 3 shows a representative immunoblot of GST fusion proteins encompassing the human, bovine, and mouse RRM probed with the anti-GST $(A)$ and human anti-La/SS-B $(B)$ sera. The human antisera were strongly positive on immunoblots of the human RRM but failed to react with either the bovine or mouse RRM-GST proteins. Control sera did not react with any of the RRM-GST proteins (data not shown).

Binding to the different human and mouse La/SS-B polypeptides by the pooled human anti-La/SS-B sera and by sera from the individual patients was then tested on ELISA (Table I). These data confirm the results of the Western blot analysis, with the human antisera reacting with the intact polypeptide

Table I. Binding of Human Anti-La/SSB-Positive Sera to Recombinant Human and Mouse La/SSB Proteins by ELISA

\begin{tabular}{|c|c|c|c|c|c|c|c|c|}
\hline \multirow[b]{2}{*}{ Patients } & \multicolumn{8}{|c|}{ Binding (expressed as $\mathrm{OD}_{40} \mathrm{~nm}$ ) to } \\
\hline & $\mathrm{h}^{*} \mathrm{LaA}$ & $\mathbf{m}^{\ddagger} \mathbf{L a A}$ & $\mathrm{hLaC}$ & $\mathrm{mLaC}$ & hLaL2/3 & $\mathrm{mLaL} 2 / 3$ & hFL-La & mFL-La \\
\hline Pool & 1.44 & 1.56 & 1.87 & 0.15 & 0.70 & 0.05 & 1.77 & 1.37 \\
\hline 1 & 0.42 & 0.40 & 1.54 & 0.09 & 1.46 & 0.06 & 1.60 & 1.10 \\
\hline 2 & 0.37 & 0.34 & 1.63 & 0.02 & 0.04 & 0.03 & 1.70 & 1.01 \\
\hline 3 & 0.63 & 0.57 & 1.75 & 0.02 & 0.06 & 0.06 & $>2$ & 1.39 \\
\hline 4 & 0.58 & 0.56 & 1.57 & 0.01 & 0.83 & 0.04 & 1.81 & 1.12 \\
\hline 5 & 0.64 & 0.57 & 1.56 & 0.01 & 1.93 & 0.06 & 1.68 & 1.07 \\
\hline 6 & 0.44 & 0.46 & 0.97 & 0.05 & 0.05 & 0.05 & 1.29 & 0.74 \\
\hline 7 & 0.28 & 0.44 & 1.75 & 0.06 & 0.18 & 0.05 & 1.84 & 0.86 \\
\hline 8 & 1.07 & 0.95 & $>2$ & 0.09 & 0.11 & 0.04 & $>2$ & 1.60 \\
\hline 9 & 0.67 & 0.70 & 1.81 & 0.05 & 1.05 & 0.07 & 1.80 & 1.23 \\
\hline 10 & 1.00 & 0.92 & 1.49 & 0.09 & 0.15 & 0.04 & 1.64 & 1.37 \\
\hline 11 & 0.37 & 0.47 & 1.47 & 0.20 & 0.26 & 0.17 & 1.65 & 1.05 \\
\hline 12 & 0.54 & 0.49 & 1.49 & 0.06 & 0.09 & 0.09 & 1.62 & 1.13 \\
\hline 13 & 1.64 & 1.25 & $>2$ & 0.16 & $>2$ & 0.19 & $>2$ & 1.82 \\
\hline 14 & 0.55 & 0.78 & 0.13 & 0.02 & 0.03 & 0.05 & 0.64 & 0.42 \\
\hline 15 & 1.47 & 1.28 & 1.82 & 0.09 & 0.71 & 0.05 & 1.87 & 1.56 \\
\hline 16 & 0.15 & 0.09 & 0.74 & 0 & 0.05 & 0.05 & 0.97 & 0.19 \\
\hline 17 & 0.44 & 0.58 & 0.07 & 0.01 & 0.31 & 0.05 & 0.52 & 0.36 \\
\hline 18 & 0.74 & 1.69 & $>2$ & 0.14 & 0.12 & 0.05 & $>2$ & 1.30 \\
\hline Mean & 0.67 & 0.70 & 1.43 & 0.07 & 0.52 & 0.07 & 1.59 & 1.07 \\
\hline SD & 0.40 & 0.39 & 0.58 & 0.06 & 0.66 & 0.04 & 0.45 & 0.44 \\
\hline \multicolumn{9}{|c|}{ Healthy controls } \\
\hline Mean & 0.05 & 0.04 & 0.05 & 0.09 & 0.03 & 0.05 & 0.08 & 0.05 \\
\hline SD & 0.03 & 0.03 & 0.02 & 0.03 & 0.02 & 0.02 & 0.03 & 0.03 \\
\hline
\end{tabular}

* Human, ${ }^{\ddagger}$ mouse. 
and $\mathrm{NH}_{2}$-terminal fragment in both the human and mouse $\mathrm{La} / \mathrm{SS}-\mathrm{B}$, but not cross-reacting on either mouse LaC or LaL2/ 3. The pooled human antisera still failed to react with mouse $\mathrm{LaC}$ despite increasing the coating concentration of antigen fivefold (data not shown). Sera from healthy controls did not react with any of the human or mouse recombinant proteins. In agreement with the immunoblot results from Fig. 3, the pooled human anti-La/SS-B sera reacted by ELISA with the human RRM ( 1.46 OD U) but did not cross-react with either the mouse RRM (0.02 OD U) or bovine RRM (0.07 OD U).

Competitive inhibition of human anti-La/SS-B antibody binding to human $\mathrm{LaC}$. The specificity of the human autoantibodies for human $\mathrm{LaC}$ (encompassing the RRM) was verified by preincubation of the pooled human anti-La/SS-B sera (at a dilution of $1: 1,000)$ with different concentrations of human and mouse LaC before testing by ELISA. Preincubation with increasing concentrations of human $\mathrm{LaC}$ but not mouse $\mathrm{LaC}$ substantially reduced the level of human anti-La/SS-B binding to human $\mathrm{LaC}$ in a dose-dependent manner (Table II), confirming that the epitope contained within the human $\mathrm{LaC}$ molecule is poorly conserved in mouse La/SS-B. Background inhibition of human $\mathrm{LaC}$ binding by addition of mouse La/SS-B antigen did not titrate, indicating nonspecific inhibition. $\mathrm{Hu}-$ man anti-La/SS-B binding was not altered by preincubation with GST alone, indicating that the anti-La/SS-B sera reacted with the La/SS-B portion of the recombinant protein and not with the GST moiety (Table II).

\section{Discussion}

A comparison of the primary sequence of human and mouse $\mathrm{La} / \mathrm{SS}-\mathrm{B}$ reveals that the $\mathrm{NH}_{2}$-terminal region containing the LaA epitope is highly conserved and that most interspecies divergence occurs in the $\mathrm{COOH}$-terminal region, which contains the human LaL2/3 epitope (Fig. 1). This region was predicted to be the most likely site for preferential binding of anti-La/SS-B autoantibodies to the human autoantigen. Sequence divergence within the $\mathrm{COOH}$-terminal half of the mole-

Table II. Inhibition of Human Anti-La/SS-B Binding to Recombinant Human La/SS-B Proteins

\begin{tabular}{|c|c|c|c|c|c|}
\hline \multirow[b]{2}{*}{ Inhibitor* } & \multicolumn{5}{|c|}{ Percent reduction in anti-La/SS-B binding to } \\
\hline & $\mu \mathrm{g} / \mathrm{ml}$ & $\mathrm{h}^{\ddagger} \mathrm{LaC}$ & $\mathrm{m}^{8} \mathrm{LaC}$ & hLaA & $\mathrm{mLaA}$ \\
\hline \multirow[t]{4}{*}{ hLaC } & 10 & 90 & - & 0 & 0 \\
\hline & 1 & 65 & - & 0 & 0 \\
\hline & 0.1 & 20 & - & 0 & 0 \\
\hline & 0.01 & 11 & - & 0 & 0 \\
\hline \multirow[t]{4}{*}{$\mathrm{mLaC}$} & 10 & 32 & - & 4 & 3 \\
\hline & 1 & 36 & - & 15 & 7 \\
\hline & 0.1 & 31 & - & 19 & 5 \\
\hline & 0.01 & 29 & - & 19 & 0 \\
\hline \multirow[t]{4}{*}{ GST } & 10 & 0 & - & NT & NT \\
\hline & 1 & 0 & - & NT & NT \\
\hline & 0.1 & 1 & - & NT & NT \\
\hline & 0.01 & 3 & - & NT & NT \\
\hline
\end{tabular}

\footnotetext{
* The pooled anti-La/SS-B sera were incubated with different concentrations of inhibitors for $1 \mathrm{~h}$ at room temperature. ${ }^{\ddagger}$ Human, ${ }^{\S}$ mouse, NT, not tested.
}

cule has also been observed for bovine La/SS-B (20) and rat La/SS-B (Dr. Michael Bachmann, personal communication). The confirmation of a human-specific epitope in the poorly conserved $\mathrm{COOH}$-terminal region implies that autoantibodies to the LaL2/3 epitope are generated by autoimmunization. The antibody binding studies described here also define a conserved $\mathrm{NH}_{2}$-terminal epitope ( $\left.\mathrm{LaA}\right)$. The LaA epitope has been shown previously to be discontinuous ( 8 ), suggesting that the $\mathrm{B}$ cell response to this region is driven by the native autoantigen and is unlikely to arise by molecular mimicry with a linear sequence of a viral gag polypeptide (6).

The lack of cross-reactivity of human anti-La/SS-B autoantibodies with the mouse and bovine RRM was unexpected because of the highly conserved nature of this region of the molecule. This observation further underlines the role of self-immunization and reinforces the notion that species-specific epitopes may be targeted in the autoimmune B cell response to nuclear autoantigens (22-24). The RRM contains nine amino acid substitutions that distinguish the human and mouse (Fig. $1 B$ ). The absence of reactivity with the bovine RRM identifies a glutamic acid at position 177, an aspartic acid at position 186, and a valine at position 156 as being specific for the human RRM (Fig. $1 B$ ). These residues are therefore of critical importance in controlling recognition of this immunodominant determinant. Analysis of autoantibody binding to deletion mutants of the human RRM suggests that as for $\operatorname{LaA}(8)$ the dominant epitope in this region is discontinuous (25). It has been shown recently that binding of antibody to an epitope present on a folded protein can be reduced or eliminated by changing single residues (26). The same phenomenon is likely to be true for the species-specific component of the B cell response to autoepitopes of La/SS-B and other nuclear autoantigens.

Our results show that the anti-La/SS-B response is characterized by a breakdown in B cell tolerance to both conserved and poorly conserved regions of $\mathrm{La} / \mathrm{SS}-\mathrm{B}$. Sera from precipitin-negative patients show a restricted $B$ cell epitope pattern with sparing of the poorly conserved $\mathrm{COOH}$-terminal epitope (21). If the precipitin-negative patient group represents an early oligoclonal response to $\mathrm{La} / \mathrm{SS}-\mathrm{B}$, then it might be inferred that antibody reactivity is directed initially to conserved epitopes on the autoantigen, with recruitment of less conserved epitopes as the autoimmune response becomes polyclonal and involves the whole molecule. Further studies, including longitudinal analysis of precipitin-negative patients and the use of animal models, will help clarify the questions of hierarchy and dominance of the autoepitopes of La/SS-B.

\section{Acknowledgments}

We thank Ms. Michelle Barker for typing the manuscript and Mrs. Catherine Keech for providing technical advice.

This work was supported by a National Health and Medical Research Council Project grant.

\section{References}

1. Chambers, J. C., D. Kenan, B. J. Martin, and J. D. Keene. 1988. Genomic structure and amino acid sequence domains of the human La autoantigen. $J$. Biol. Chem. 263:18043-18051.

2. Sturgess, A. D., M. G. Peterson, L. J. McNeilage, S. Whittingham, and R. L. Coppel. 1988. Characteristics and epitope mapping of a cloned human autoantigen La. J. Immunol. 140:2312-2318. 
3. Rauh, A. J. G., H. Hornig, and R. Luhrmann. 1988. At least 3 distinct B cell epitopes reside in the C-terminal half of La protein, as determined by a recombinant DNA approach. Eur. J. Immunol. 18:2049-2057.

4. St. Clair, E. W., D. S. Pisetsky, C. F. Reich, and J. D. Keene. 1988. Analysis of autoantibody binding to different regions of the human La antigen expressed in recombinant fusion proteins. J. Immunol. 141:4173-4180.

5. Bini, P., J.-L. Chu, C. Okolo, and K. Elkon. 1990. Analysis of autoantibodies to recombinant $\mathrm{La}(\mathrm{SS}-\mathrm{B})$ peptides in systemic lupus erythematosus and primary Sjögren's syndrome. J. Clin. Invest. 85:325-333.

6. Kohsaka, H., K. Yamamoto, H. Fujii, H. Miura, N. Miyasaka, K. Nishioka, and T. Miyamoto. 1990. Fine epitope mapping of the human SS-B/La protein. Identification of a distinct autoepitope homologous to a viral gag polyprotein. J. Clin. Invest. 85:1566-1574.

7. McNeilage, L. J., E. M. Macmillan, and S. F. Whittingham. 1990. Mapping of epitopes on the $\mathrm{La}$ (SS-B) autoantigen of primary Sjögren's syndrome: identification of a cross-reactive epitope. J. Immunol. 145:3829-3835.

8. McNeilage, L. J., K. Umapathysivam, E. Macmillan, A. Guidolin, S. Whittingham, and T. Gordon. 1992. Definition of a discontinuous immunodominant epitope at the $\mathrm{NH}_{2}$ terminus of the La/SS-B ribonucleoprotein autoantigen. $J$. Clin. Invest. 89:1652-1656.

9. Stefano, J. E. 1984. Purified lupus antigen La recognises an oligouridylate stretch common to the $3^{\prime}$ termini of RNA polymerase III transcripts. Cell. 36:145-154.

10. Pruijn, G. J. M., R. L. Slobbe, and W. J. van Venrooij. 1991. Analysis of protein-RNA interactions within Ro ribonucleoprotein complexes. Nucleic Acids Res. 19:5173-5180.

11. Gordon, T. P., M. Greer, P. Reynolds, A. Guidolin, and L. J. McNeilage. 1991. Estimation of amounts of anti-La(SS-B) antibody directed against immunodominant epitopes of the La(SS-B) autoantigen. Clin. Exp. Immunol. 85:402406.

12. Reveille, J. D., M. J. Macleod, K. Whittington, and F. C. Arnett. 1991. Specific amino acid residues in the second hypervariable region of HLA-DQA and DQB1 chain genes promote the Ro(SS-A)/La(SS-B) autoantibody responses. J. Immunol. 146:3871-3876.

13. Chan, E. K. L., and E. M. Tan. 1987. Human autoantibody-reactive epitopes of SS-B/La are highly conserved in comparison with epitopes recognized by murine monoclonal antibodies. J. Exp. Med. 166:1627-1460.
14. Hardin, J. A. 1986. The lupus autoantigens and the pathogenesis of systemic lupus erythematosus. Arthritis Rheum. 29:457-460.

15. Tan, E. M. 1989. Antinuclear antibodies: diagnostic markers for autoimmune diseases and probes for cell biology. Adv. Immunol. 44:93-151.

16. Topfer, F., T. Gordon, and J. McCluskey. 1993. Characterisation of the mouse autoantigen La(SS-B). Identification of conserved RNA-binding motifs, a putative ATP binding site and reactivity of recombinant protein with poly(U) and human autoantibodies. J. Immunol. 150:3091-3100.

17. Pearson, W. R., and D. J. Lipman. 1988. Improved tools for biological sequence analysis. Proc. Natl. Acad. Sci. USA. 85:2444-2448.

18. Saiki, R. K., D. H. Gelfand, S. Stoffel, S. J. Scharf, G. T. Hora, K. B. Mullis, and H. A. Erlich. 1988. Primer-directed enzymatic amplification of DNA with a thermostable DNA polymerase. Science (Wash. DC). 239:487-491.

19. Gordon, T., B. Grove, J. C. Loftus, T. O'Toole, R. McMillan, J. Lindstrom, and M. H. Ginsberg. 1992. Molecular cloning and preliminary characterization of a novel cytoplasmic antigen recognized by myasthenia gravis sera. $J$. Clin. Invest. 90:992-999.

20. Chan, E. K. L., K. F. Sullivan, and E. M. Tan. 1989. Ribonucleoprotein SS-B/La belongs to a protein family with consensus sequences for RNA binding. Nucleic Acids Res. 17:2233-2244.

21. Gordon, T., C. Mavrangelos, and J. McCluskey. 1992. Restricted epitope recognition by precipitin-negative anti-La/SS-B-positive sera. Arthritis Rheum. 35:663-666

22. Reichlin, M., M. Rader, and J. B. Harley. 1989. Autoimmune response to the Ro/SS-A particle is directed to the human antigen. Clin. Exp. Immunol. 76:373-337.

23. Boire, G., and J. Craft. 1989. Biochemical and immunological heterogeneity of the Ro ribonucleoprotein particles. Analysis with sera specific for the Ro ${ }^{\text {hYs }}$ particle. J. Clin. Invest. 84:270-279.

24. Porges, A. J., T. Ng, and W. H. Reeves. 1990. Antigenic determinants of the $\mathrm{Ku}(\mathrm{p} 70 / \mathrm{p} 80)$ autoantigen are poorly conserved between species. $J$. Immunol. 145:4222-4228.

25. Rischmueller, M., L. J. McNeilage, K. Umapathysivam, and T. Gordon. 1992. Reactivity of human autoantibodies to the RNA binding domain of the La/SS-B ribonucleoprotein autoantigen. Aust. N.Z.J. Med. 22:183a. (Abstr).

26. Alexander, H. S., E. D. Alexander, J. A. Getzoff, H. M. Tainer, H. M. Geysen, and R. A. Lerner. 1992. Altering the antigenicity of proteins. Proc. Natl. Acad. Sci. USA. 89:3352-3356. 